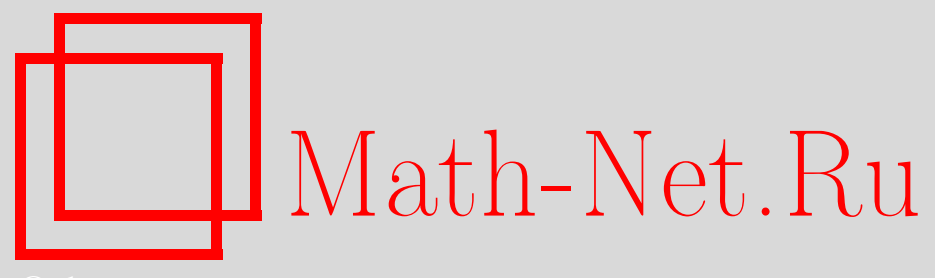

В. Б. Алексеев, Минимальные расширения с простым умножением для алгебры матриц второго порядка, Дискрет. матем., 1997, том 9, выпуск 1, 71-82

DOI: https://doi.org/10.4213/dm449

Использование Общероссийского математического портала Math-Net.Ru подразумевает, что вы прочитали и согласны с пользовательским соглашением http: //www.mathnet.ru/rus/agreement

Параметры загрузки:

IP : 3.82 .47 .9

26 апреля 2023 г., 18:33:46

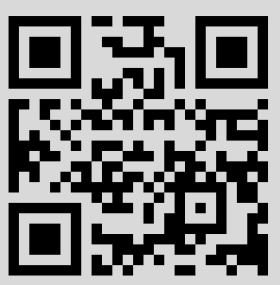




\title{
Минимальные расширения с простым умножением для алгебры матриц второго поря,дка
}

(C) 1997 г. В. Б. Алексеев

\begin{abstract}
В работе показано, что результат Штрассена о существовании билинейного алгоритма с 7 умножениями для умножения матриц порядка 2 допускает простую трактовку: существует 7-мерная алгебра с простым умножением, являющаяся расширением алгебры матриц порядка 2. Доказано, что существуют ровно 3 такие 7 -мерные алгебры.

Работа выполнена при поддержке Российского фонда фундаментальных исследований, проект 94-01-01206.
\end{abstract}

В [1] введен класс алгебр с простым умножением и показано, что результаты о существовании быстрых билинейных алгоритмов для умножения комплексных чисел и кватернионов могут быть представлены просто как вложение этих алгебр в алгебры с простым умножением. В частности, там построены все трехмерные алгебры с простым умножением над полем действительных чисел, являющиеся расширением алгебры комплексных чисел. Алгебры с простым умножением были определены следующим образом.

Определение 1. Пусть $K$ - некоторое поле, $V$ - векторное пространство над $K$ с дополнительной операцией $x \cdot y$ умножения в $V$, удовлетворяющей свойству линейности по каждому из аргументов. Тогда $V$ называется алгеброй над полем $K$.

Определение 2. Пусть $V$ - алгебра на полем $K$. Тогда $V$ будет называться алгеброй с простым умножением, если существует такой базис $e_{1}, e_{2}, \ldots, e_{n}$ в $V$ и существует такая подстановка $\sigma$ на множестве $\{1,2, \ldots, n\}$, что $e_{i} e_{j}=0$ при $j \neq \sigma(i)$.

Таким образом, умножение в $n$-мерной алгебре $V$ с простым умножением задается $n$ равенствами

$$
e_{i} e_{\sigma(i)}=f_{i}=\sum_{k=1}^{n} \gamma_{i k} e_{k}, \quad i=1,2, \ldots, n
$$

и условием, что все остальные произведения базисных векторов равны нулю. 


\section{Таблица 1.}

\begin{tabular}{l|rrrrrrr} 
& $e_{0}$ & $e_{1}$ & $e_{2}$ & $e_{3}$ & $e_{4}$ & $e_{5}$ & $e_{6}$ \\
\hline$e_{0} \cdot e_{0}$ & 2 & -1 & -1 & -1 & -1 & -1 & -1 \\
$e_{2} \cdot e_{3}$ & -1 & 1 & 0 & 0 & 1 & 1 & 0 \\
$e_{3} \cdot e_{1}$ & -1 & 0 & 1 & 0 & 1 & 0 & 1 \\
$e_{1} \cdot e_{2}$ & -1 & 0 & 0 & 1 & 0 & 1 & 1 \\
$e_{5} \cdot e_{6}$ & -1 & 1 & 1 & 0 & 1 & 0 & 0 \\
$e_{6} \cdot e_{4}$ & -1 & 1 & 0 & 1 & 0 & 1 & 0 \\
$e_{4} \cdot e_{5}$ & -1 & 0 & 1 & 1 & 0 & 0 & 1
\end{tabular}

Пусть $M_{2}$ - алгебра матриц второго порядка над полем действительных чисел, и пусть $e_{i j}, i=1,2, j=1,2$, обозначает матрицу, у которой элемент в строке $i$ и столбце $j$ равен единице, а остальные элементы равны нулю. Тогда $e_{11}, e_{12}, e_{21}, e_{22}$ - базис в $M_{2}$, и таблица умножения в $M_{2}$ задается равенствами

$$
e_{i j} e_{k m}=\delta_{j k} e_{i m},
$$

где $\delta_{j k}$ - дельта Кронекера. Обычный алгоритм умножения двух матриц порядка 2 требует 8 умножений их элементов. В [2] для умножения двух матриц порядка 2 был предложен билинейный алгоритм (определение см. ниже) сложности 7. Позднее было доказано, что это оптимальный по сложности билинейный алгоритм [3]. Для умножения матриц порядка $n \geqslant 3$ оптимальная билинейная сложность неизвестна. Так, для $n=3$ известно только, что она заключена между 17 и 23 [4]. В связи с этим представляется важным исследовать свойства оптимальных алгоритмов для умножения матриц порядка 2 с тем, чтобы попытаться обобщить их на большие $n$.

Мы покажем здесь, что результат о существовании билинейного алгоритма сложности 7 для умножения матриц порядка 2 допускает простую трактовку, а именно: существует 7-мерная алгебра с простым умножением, являющаяся расширением алгебры матриц порядка 2. Заметим, что, поскольку билинейная сложность умножения матриц порядка 2 равна 7 , по лемме 3 из [1] не существует 6 -мерной алгебры с такими же свойствами. Основной результат мы получим в следующей более сильной форме.

Теорема 1. Существуют ровно 3 с точностью до изоморфизма 7-мерные алгебрь с простым умножением над полем действительных чисел, являющиеся расширением алгебры матриц второго порядка, а именно, алгебры, ненулевые произведения базисных әлементов в которых заданы в табличах 1, 3, 4.

Доказательство. Пусть $A_{m}, B_{m}, C_{m}, m=0,1, \ldots, 6$, - матрицы порядка $2 \mathrm{c}$ действительными коэффициентами. Будем говорить, что эти матрицы задают билинейный алгоритм сложности 7 для умножения двух матриц $P=\left\|p_{i j}\right\|$ и 


\section{Таблица 2.}

\begin{tabular}{l|rrrrrrr} 
& $e_{0}$ & $e_{1}$ & $e_{2}$ & $e_{3}$ & $e_{4}$ & $e_{5}$ & $e_{6}$ \\
\hline$e_{0} \cdot e_{0}$ & 0 & 1 & 0 & -1 & 1 & 0 & -1 \\
$e_{1} \cdot e_{5}$ & -1 & 0 & 0 & 1 & 0 & 1 & 1 \\
$e_{2} \cdot e_{6}$ & 1 & -1 & -1 & 0 & -1 & 0 & 0 \\
$e_{3} \cdot e_{4}$ & 0 & 0 & 0 & 1 & -1 & 0 & 0 \\
$e_{4} \cdot e_{2}$ & -1 & 0 & 1 & 1 & 0 & 0 & 1 \\
$e_{5} \cdot e_{3}$ & 1 & -1 & 0 & 0 & -1 & -1 & 0 \\
$e_{6} \cdot e_{1}$ & 0 & -1 & 0 & 0 & 0 & 0 & 1
\end{tabular}

\section{Таблица 3.}

\begin{tabular}{l|rrrrrrr} 
& $e_{0}$ & $e_{1}$ & $e_{2}$ & $e_{3}$ & $e_{4}$ & $e_{5}$ & $e_{6}$ \\
\hline$e_{0} \cdot e_{0}$ & 0 & 1 & 0 & 1 & 1 & 0 & 1 \\
$e_{5} \cdot e_{3}$ & 1 & -1 & 0 & 0 & -1 & 1 & 0 \\
$e_{3} \cdot e_{4}$ & 0 & 0 & 0 & 1 & 1 & 0 & 0 \\
$e_{4} \cdot e_{2}$ & 1 & 0 & 1 & 1 & 0 & 0 & 1 \\
$e_{2} \cdot e_{6}$ & 1 & -1 & 1 & 0 & -1 & 0 & 0 \\
$e_{6} \cdot e_{1}$ & 0 & 1 & 0 & 0 & 0 & 0 & 1 \\
$e_{1} \cdot e_{5}$ & 1 & 0 & 0 & 1 & 0 & 1 & 1
\end{tabular}

$Q=\left\|q_{i j}\right\|$ порядка 2, если из равенств при $m=0,1, \ldots, 6$

$$
\begin{aligned}
& F_{m}^{\prime}=\sum_{i=1^{2}} \sum_{j=1}^{2} a_{i j}^{m} p_{i j}, \quad F_{m}^{\prime \prime}=\sum_{i=1}^{2} \sum_{j=1}^{2} b_{i j}^{m} q_{i j} \\
& r_{i j}=\sum_{m=0}^{6} c_{j i}^{m} F_{m}, \quad F_{m}=F_{m}^{\prime} F_{m}^{\prime \prime}, \quad i, j=1,2,
\end{aligned}
$$

\section{Таблица 4.}

\begin{tabular}{l|rrrrrrr} 
& $e_{0}$ & $e_{1}$ & $e_{2}$ & $e_{3}$ & $e_{4}$ & $e_{5}$ & $e_{6}$ \\
\hline$e_{0} \cdot e_{0}$ & 1 & 0 & 0 & 0 & -1 & -1 & -1 \\
$e_{1} \cdot e_{1}$ & 0 & -1 & 0 & 0 & 0 & 0 & 1 \\
$e_{2} \cdot e_{2}$ & 0 & 0 & -1 & 0 & 0 & 1 & 0 \\
$e_{3} \cdot e_{3}$ & 0 & 0 & 0 & -1 & 1 & 0 & 0 \\
$e_{6} \cdot e_{5}$ & -1 & 0 & 0 & 1 & 0 & 1 & 1 \\
$e_{4} \cdot e_{6}$ & -1 & 0 & 1 & 0 & 1 & 0 & 1 \\
$e_{5} \cdot e_{4}$ & -1 & 1 & 0 & 0 & 1 & 1 & 0
\end{tabular}


следует, что $R=\left\|r_{i j}\right\|=P Q$. Заметим, что при вычислении $r_{i j}$ используются матрицы, транспонированные к $C_{m}$.

Рассмотрим следующие 7 матриц порядка 2:

$$
\begin{gathered}
S_{0}=E=\left\|\begin{array}{ll}
1 & 0 \\
0 & 1
\end{array}\right\|, \quad S_{1}=\left\|\begin{array}{rr}
0 & 0 \\
-1 & -1
\end{array}\right\|, \quad S_{2}=\left\|\begin{array}{rr}
-1 & 0 \\
0 & 0
\end{array}\right\|, \quad S_{3}=\left\|\begin{array}{rr}
0 & 1 \\
0 & -1
\end{array}\right\|, \\
S_{4}=\left\|\begin{array}{rr}
-1 & -1 \\
0 & 0
\end{array}\right\|, \quad S_{5}=\left\|\begin{array}{rr}
0 & 0 \\
0 & -1
\end{array}\right\|, \quad S_{6}=\left\|\begin{array}{rr}
-1 & 0 \\
1 & 0
\end{array}\right\| .
\end{gathered}
$$

Пусть $\rho$ - подстановка на множестве $\{0,1, \ldots, 6\}$, следующим образом распадающаяся на циклы $\rho=(0)(123)(456)$. Следующее утверждение легко проверяется.

Лемма 1 ([2]). Пусть

$$
A_{m}=S_{m}, \quad B_{m}=S_{\rho(m)}, \quad C_{m}=S_{\rho_{(m)}^{2}}, \quad m=0,1, \ldots, 6 .
$$

Тогда матрицы $A_{m}, B_{m}, C_{m}, m=0,1, \ldots, 6$, задают билинейный алгоритм сложности 7 для умнохсения двух матриц.

На матрицы (2) мы будем ссылаться как на матрицы Штрассена. Следующая лемма описывает все билинейные алгоритмы сложности 7 для умножения матриц порядка 2.

Лемма 2 ([3]). Пусть $A_{m}, B_{m}, C_{m}, m=0,1, \ldots, 6$,- матрицы порядка 2 с действительными әлементами, задающие билинейный алгоритм для умножения двух матрич порядка 2 над полем действительных чисел.

Тогда с точностью до одновременной перенумерачии всех матрич $A_{m}, B_{m}, C_{m}$ существуют невырожденные матрицы $X, Y, Z$ порядка 2 и такие константы $\lambda_{m}^{a}, \lambda_{m}^{b}, \lambda_{m}^{c}, m=0,1, \ldots, 6$, ито

$$
\begin{gathered}
A_{m}=\lambda_{m}^{a} X S_{m} Y^{-1}, \quad B_{m}=\lambda_{m}^{b} Y S_{\rho(m)} Z^{-1}, \\
C_{m}=\lambda_{m}^{c} Z S_{\rho^{2}(m)} X^{-1}, \quad \lambda_{m}^{a} \lambda_{m}^{b} \lambda_{m}^{c}=1, \quad m=0,1, \ldots, 6,
\end{gathered}
$$

где $S_{m}$ - матрицы Штрассена (2) и $\rho=(0)(123)(456)$.

Установим связь между билинейными алгоритмами сложности 7 для умножения матриц порядка 2 и 7-мерными алгебрами с простым умножением, содержащими подалгебры, изоморфные алгебре матриц порядка 2.

Лемма 3. Пусть $A_{m}, B_{m}, C_{m}, m=0,1, \ldots, 6$ - матрицы порядка 2 с действительными әлементами, задающие билинейный алгоритм для умножсения двух матрич порядка 2. Пусть $\sigma$ - подстановка на множестве $\{0,1, \ldots, 6\}$ такая, что $B_{m}=A_{\sigma(m)}$. Положсим $\gamma_{i k}=\operatorname{Tr}\left(A_{k} C_{i}\right)$, где $\operatorname{Tr}-$ след матрицы. Тогда 7-мерная алгебра $V$ с простым умножением

$$
e_{i} e_{\sigma(i)}=\sum_{k=0}^{6} \gamma_{i k} e_{k}, \quad i=0,1, \ldots, 6, \quad e_{i} e_{j}=0, \quad j \neq i,
$$

содерэісит подалгебру, изоморфную алгебре матрич, порядка 2 над полем действительных чисел. 
Доказательство. Рассмотрим вложение $\varphi$ алгебры $M_{2}$ матриц порядка 2 в алгебру $V$, при котором произвольная матрица $P=\left\|p_{i j}\right\|$ переходит в вектор

$$
\sum_{m=0}^{6} \alpha_{m} e_{m}
$$

такой, что

$$
\alpha_{m}=\sum_{i=1}^{2} \sum_{j=1}^{2} a_{i j}^{m} p_{i j}, \quad m=0,1, \ldots, 6,
$$

где $a_{i j}^{m}$ - элементы матрицы $A_{m}$. Очевидно, это отображение изоморфно относительно сложения и умножения на действительную константу. Пусть $P, Q, R-$ матрицы порядка 2 и $R=P Q$. Так как матрицы $A_{m}, B_{m}, C_{m}$ задают билинейный алгоритм для умножения матриц порядка 2 , то для $P, Q, R$ выполняются равенства (1). Покажем, что в алгебре $V$ справедливо равенство $\varphi(R)=\varphi(P) \varphi(Q)$. Ясно, что

$$
\begin{aligned}
\varphi(P) & =\sum_{m-0}^{6} \sum_{i=1}^{2} \sum_{j=1}^{2} a_{i j}^{m} p_{i j} e_{m}=\sum_{m=0}^{6} F_{m}^{\prime} e_{m} \\
\varphi(Q) & =\sum_{m=0}^{6} \sum_{i=1}^{2} \sum_{j=1}^{2} a_{i j}^{m} q_{i j} e_{m} \sum_{m=0}^{6} \sum_{i=1}^{2} \sum_{j=1}^{2} a_{i j}^{\sigma(m)} q_{i j} e_{m} \\
& =\sum_{m=0}^{6} \sum_{i=1}^{2} \sum_{j=1}^{2} b_{i j}^{m} q_{i j} e_{\sigma(m)}=\sum_{m=0}^{6} F_{m}^{\prime \prime} e_{\sigma(m)}
\end{aligned}
$$

Отсюда, учитывая (1), получаем, что

$$
\begin{aligned}
\varphi(P) \varphi(Q) & =\sum_{m=0}^{6} F^{\prime} F^{\prime \prime} e_{m} e_{\sigma(m)}=\sum_{k=0}^{6} \sum_{m=0}^{6} \gamma_{m k} F_{m} e_{k} \\
& =\sum_{k=0}^{6} \sum_{m=0}^{6} \sum_{i=1}^{2} \sum_{j=1}^{2} a_{i j}^{k} c_{j i}^{m} F_{m} e_{k}=\sum_{k=0}^{6} \sum_{i=1}^{2} \sum_{j=1}^{2} a_{i j}^{k} r_{i j} e_{k}=\varphi(R) .
\end{aligned}
$$

Лемма доказана.

Лемма 4. Пусть $A_{m}, B_{m}, C_{m}, m=0,1, \ldots, 6$, - произвольные матрицы порядка 2 с действительными элементами такие, что (1) задает билинейный алгоритм для умножсения матрич порядка 2. Тогда набор матрич $C_{m}$ однозначно определяется по наборам матрич $A_{m} u B_{m}$.

Доказательство. Пусть наборы матриц $A_{m}$ и $B_{m}, m=0,1, \ldots, 6$, фиксированы. Тогда фиксированы билинейные формы $F_{m}$ в (1) от переменных $p_{i j}$ и $q_{i j}$. Если бы формы $F_{m}$ были линейно зависимы, то одну из них можно было бы выразить через остальные и получить билинейный алгоритм для умножения матриц порядка 2 сложности 6. Однако, такого алгоритма не существует [3], и, следовательно, формы $F_{m}$ линейно независимы. Так как $r_{i j}$ - фиксированные билинейные формы от переменных $p_{i j}$ и $q_{i j}$, коэффициенты $c_{j i}^{m}$ в (1) однозначно определяются. Лемма доказана. 
Лемма 5. Пусть $V$ - алгебра с простым умножсением

$$
e_{i} e_{\sigma(i)}=\sum_{k=0}^{6} \gamma_{i k} e_{k}, \quad i=0,1, \ldots, 6, \quad e_{i} e_{j}=0, \quad j \neq \sigma(i),
$$

над полем действительных чисел, содержащая подалгебру, изоморфную алгебре $M_{2}$ матрич порядка 2. Пусть отображсение

$$
\varphi(P)=\sum_{m=0}^{6} \sum_{i=1}^{2} \sum_{j=1}^{2} a_{i j}^{m} p_{i j} e_{m}
$$

где $P=\left\|p_{i j}\right\|-$ произвольная матрица порядка 2 , яөляется изоморфным вложсением алгебры $M_{2}$ в $V$. Положим

$$
A_{m}=\left\|a_{i j}^{m}\right\|, \quad B_{m}=\left\|a_{i j}^{\sigma(m)}\right\|, \quad m=0,1, \ldots, 6 .
$$

Тогда существуют матрицы $C_{m}$ порядка 2 такие, что матрицы $A_{m}, B_{m}, C_{m}$ задают билинейный алгоритм для умножсения матрич порядка $2 u \gamma_{m k}=\operatorname{Tr}\left(A_{k} C_{m}\right)$, где $\operatorname{Tr}-$ след матрицы.

Доказательство. Пусть $\psi$ - произвольное линейное отображение $V$ в $M_{2}$ такое, что $\psi(\varphi(P))=P$ для любой матрицы $P$. Пусть $\psi$ задается равенствами

$$
\psi\left(\sum_{k=0}^{6} \alpha_{k} e_{k}\right)=\left\|t_{i j}\right\|
$$

где

$$
t_{i j}=\sum_{k=0}^{6} h_{i j}^{k} \alpha_{k}, \quad i=1,2, \quad j=1,2 .
$$

Пусть $P=\left\|p_{i j}\right\|, Q=\left\|q_{i j}\right\|$ - произвольные матрицы порядка 2 и $R=\left\|r_{i j}\right\|=P Q$. Тогда

$$
\psi(\varphi(P) \varphi(Q))=\psi(\varphi(R))=R
$$

Положим

$$
F_{m}=F_{m}^{\prime} F_{m}^{\prime \prime}
$$

где

$$
F_{m}^{\prime}=\sum_{i=1}^{2} \sum_{j=1}^{2} a_{i j}^{m} p_{i j}, \quad F_{m}^{\prime \prime}=\sum_{i=1}^{2} \sum_{j=1}^{2} a_{i j}^{\sigma(m)} q_{i j}
$$

Тогда

$$
\begin{gathered}
\varphi(P)=\sum_{m=0}^{6} F_{m}^{\prime} e_{m}, \quad \varphi(Q)=\sum_{m=0}^{6} F_{m}^{\prime \prime} e_{\sigma(m)} \\
\varphi(P) \varphi(Q)=\sum_{m=0}^{6} F_{m}^{\prime} F_{m}^{\prime \prime} \sum_{k=0}^{6} \gamma_{m k} e_{k}=\sum_{k=0}^{6} \sum_{m=0}^{6} \gamma_{m k} F_{m} e_{k} .
\end{gathered}
$$


Из равенства $\psi(\varphi(P) \varphi(Q))=R$ получаем, что

$$
r_{i j}=\sum_{k=0}^{6} h_{i j}^{k} \sum_{m=0}^{6} \gamma_{m k} F_{m}=\sum_{m=0}^{6} \sum_{k=0}^{6} h_{i j}^{k} \gamma_{m k} F_{m}
$$

Положим

$$
c_{j i}^{m}=\sum_{k=0}^{6} h_{i j}^{k} \gamma_{m k} .
$$

Тогда, согласно (3), (4) и (5) матрицы $A_{m}, B_{m}, C_{m}=\left\|c_{i j}^{m}\right\|$ задают билинейный алгоритм умножения двух матриц порядка 2 .

Пусть $V_{1}$ - образ алгебры $M_{2}$ при вложении $\varphi$. Рассмотрим векторы

$$
d_{m}=\sum_{k=0}^{6} \gamma_{m k} e_{k}, \quad m=0,1, \ldots, 6 .
$$

Равенство (5) означает, что $C_{m}^{T}=\psi\left(d_{m}\right)$, где $T$ означает транспонирование. Так как матрицы $A_{m}, B_{m}$ фиксированы, по лемме 4 матрицы $C_{m}$ определяются однозначно, то есть $\psi\left(d_{m}\right)$ не зависит от выбора отображения $\psi$. Это означает, что $d_{m} \in V_{1}=\varphi\left(M_{2}\right)$. (В частности, отсюда следует, что $V^{2}$ изоморфно $M_{2}$ ). На $V_{1}$ отображение $\psi$ обратно к $\varphi$, поэтому $\varphi\left(\psi\left(d_{m}\right)\right)=d_{m}$ при всех $m=0,1, \ldots, 6$. Поскольку $\psi\left(d_{m}\right)=C_{m}^{T}$, получаем, что

$$
\sum_{k=0}^{6} \sum_{i=1}^{2} \sum_{j=1}^{2}\left(a_{i j}^{k} c_{j i}^{m}\right) e_{k}=\sum_{k=0}^{6} \gamma_{m k} e_{k}
$$

Отсюда, $\gamma_{m k}=\operatorname{Tr}\left(A_{k} C_{m}\right)$. Лемма доказана.

Лемма 6. Все 7-мерные алгебры над полем действительных иисел с простым умножением

$$
e_{i} e_{\sigma(i)}=\sum_{k=0}^{6} \gamma_{i k} e_{k}, \quad e_{i} e_{j}=0, \quad j \neq \sigma(i),
$$

являющиеся расширением алгебры $M_{2}$ матрич порядка 2, могут быть получень по формулам

$$
\gamma_{i k}=\operatorname{Tr}\left(S_{k} H^{-1} S_{\rho^{2}(i)}\right), \quad \sigma(i)=\tau^{-1}(\rho(i)),
$$

где $\rho=(0)(123)(456)$, а $H-$ произвольная матрица порядка 2, удовлетворяющая условию

$$
H S_{m} H^{-1}=S_{\tau(m)}
$$

для матриц Штрассена $S_{0}, S_{1}, \ldots, S_{6}$ из (2) и некоторой подстановки $\tau$ на множсестве $\{0,1, \ldots, 6\}$. 
Доказательство. Леммы 3 и 5 показывают, что все 7-мерные алгебры с простым умножением над полем действительных чисел, являющиеся расширением алгебры $M_{2}$ матриц порядка 2 - это в точности те алгебры, которые получаются так, как указано в лемме 3 , из наборов матриц $A_{m}, B_{m}, C_{m}, m=0,1, \ldots, 6$, задающих билинейный алгоритм умножения матриц порядка 2 , с дополнительным условием, что набор матриц $B_{m}$ является перестановкой набора матриц $A_{m}$.

Рассмотрим все такие наборы матриц $A_{m}, B_{m}, C_{m}$. По лемме 2 все они задаются равенствами

$$
\begin{gathered}
A_{m}=\lambda_{m}^{a} X S_{m} Y^{-1}, \quad B_{m}=\lambda_{m}^{b} Y S_{\rho(m)} Z^{-1}, \\
C_{m}=\lambda_{m}^{c} Z S_{\rho^{2}(m)} X^{-1}, \quad \lambda_{m}^{a} \lambda_{m}^{b} \lambda_{m}^{c}=1, \quad m=0,1, \ldots, 6,
\end{gathered}
$$

где $S_{i}, i=0,1, \ldots, 6,-$ матрицы Штрассена, $X, Y, Z$ - произвольные невырожденные матрицы и $\rho=(0)(123)(456)$. Сделаем замену

$$
\lambda_{0}^{a} X \rightarrow X, \quad Z / \lambda_{0}^{b} \rightarrow Z
$$

Тогда коэффициенты $\lambda_{m}^{a}$ поделятся на $\lambda_{0}^{a}, \lambda_{m}^{b}$ поделятся на $\lambda_{0}^{b}$ и $\lambda_{m}^{c}$ поделятся на $\lambda_{0}^{c}=1 /\left(\lambda_{0}^{a} \lambda_{0}^{b}\right)$. Мы будем считать, что в (9) уже произведено такое преобразование. Тогда $\lambda_{0}^{a}=\lambda_{0}^{b}=\lambda_{0}^{c}=1$.

Имеется дополнительное условие, состоящее в том, что $B_{m}=A_{\sigma(m)}$ для некоторой подстановки $\sigma$. Заметим, что у всех матриц Штрассена $S_{i}, i=1, \ldots, 6$, ранг равен 1 , кроме матрицы $S_{0}=E$. Поэтому ранг матриц $A_{1}, \ldots, A_{6}$, $B_{1}, \ldots, B_{6}$ равен 1 , a $\operatorname{rank} A_{0}=\operatorname{rank} B_{0}=2$. Поэтому обязательно $\sigma(0)=0$ и

$$
\lambda_{0}^{a} X S_{0} Y^{-1}=\lambda_{0}^{b} Y S_{0} Z^{-1}
$$

Так как $\lambda_{0}^{a}=\lambda_{0}^{b}=1$, то $X Y^{-1}=Y Z^{-1}$. Отсюда $Z=Y X^{-1} Y$. Положим $\rho\left(\sigma^{-1}(m)\right)=\tau(m)$. Так как $A_{m}=B_{\sigma^{-1}(m)}$, справедливы равенства

$$
\begin{gathered}
\lambda_{m}^{a} X S_{m} Y^{-1}=\lambda_{\sigma^{-1}(m)}^{b} Y S_{\tau(m)} Z^{-1}, \\
S_{\tau(m)}=\frac{\lambda_{m}^{a}}{\lambda_{\sigma^{-1}(m)}^{b}} Y^{-1} X S_{m} Y^{-1} Z=\frac{\lambda_{m}^{a}}{\lambda_{\sigma^{-1}(m)}^{b}} Y^{-1} X S_{m} X^{-1} Y
\end{gathered}
$$

для всех $m$. Положим $H=Y^{-1} X$. Тогда

$$
S_{\tau(m)}=\frac{\lambda_{m}^{a}}{\lambda_{\sigma^{-1}(m)}^{b}} H S_{m} H^{-1} .
$$

Нетрудно видеть, что собственные значения каждой матрицы $S_{m}$ равны 0 и -1 . Тогда у матрицы $S_{\tau(m)}$ собственные числа равны 0 и $-\lambda_{m}^{a} / \lambda_{\sigma^{-1}(m)}^{b}$. Отсюда,

$$
\lambda_{m}^{b}=\lambda_{\sigma(m)}^{a}, \quad S_{\tau(m)}=H S_{m} H^{-1}, \quad m=0,1, \ldots, 6 .
$$

При этом для соответствующей 7-мерной алгебры, согласно лемме 3 , справедливы равенства

$$
e_{i} e_{\sigma(i)}=\sum_{k=0}^{6} \gamma_{i k} e_{k}
$$


где

$$
\gamma_{i k}=\operatorname{Tr}\left(A_{k} C_{i}\right)=\lambda_{k}^{a} \lambda_{i}^{c} \operatorname{Tr}\left(X S_{k} Y^{-1} Z S_{\rho_{(i)}^{2}} X^{-1}\right)=\lambda_{k}^{a} \lambda_{i}^{c} \operatorname{Tr}\left(S_{k} H^{-1} S_{\rho_{(i)}^{2}}\right) .
$$

Приведем эту алгебру к более удобной форме, избавившись от констант. Заменим $e_{i}$ на $e_{i}^{\prime}=\lambda_{i}^{a} e_{i}$. Тогда таблица умножения в новом базисе получается умножением $\gamma_{i k}$ на

$$
\frac{\lambda_{i}^{a} \lambda_{\sigma(m)}^{a}}{\lambda_{k}^{a}}=\frac{\lambda_{i}^{a} \lambda_{i}^{b}}{\lambda_{k}^{a}}=\frac{1}{\lambda_{i}^{c} \lambda_{k}^{a}}
$$

Следовательно, после такой замены

$$
\gamma_{i k}=\operatorname{Tr}\left(S_{k} H^{-1} S_{\rho_{(i)}^{2}}\right)
$$

и все условия леммы выполняются.

С другой стороны, пусть $H-$ произвольная матрица такая, что $H S_{m} H^{-1}=$ $S_{\tau(m)}$ для некоторой подстановки $\tau$. Взяв $X=H, Y=E, Z=H^{-1}$ и положив все $\lambda$ равными единице, получим, что $X S_{m} Y^{-1}=Y S_{\tau(m)} Z^{-1}$ и $B_{m}=A_{\sigma(m)}$, где $\sigma(m)=\tau^{-1}(\rho(m))$. При этом $Y^{-1} X=H$, то есть такая матрица $H$ может получиться при рассмотрении различных наборов матриц $A_{m}, B_{m}, C_{m}$. Лемма полностью доказана.

Лемма 7. Пусть $w_{1}=(1,0), w_{2}=(0,-1), w_{3}=(-1,1)$. Тогда в лемме 6 достаточно рассматривать только матрицы $H$, для которых $\left\{H w_{1}, H w_{2}, H w_{3}\right\}=$ $\left\{w_{1}, w_{2}, w_{3}\right\}$.

Доказательство. Так как $H E H^{-1}=E$, в (8) всегда $\tau(0)=0$. Рассмотрим собственные значения и собственные векторы матриц $S_{1}, \ldots, S_{6}$. Нетрудно видеть, что у каждой из этих матриц одно собственное значение равно нулю и одно равно -1. При этом собственными векторами каждой матрицы являются два вектора из списка $w_{1}=(1,0), w_{2}=(0,-1), w_{3}=(-1,1)$. Пусть $S_{m} x=\lambda x$ для вектор-столбца $x$. Если выполняется (8), то $S_{\tau(m)} H x=\lambda H x$. Следовательно, собственные векторы матрицы $S_{m}$ под действием $H$ переходят в собственные векторы матрицы $S_{\tau(m)}$. Таким образом, если выполняется $(8)$, то

$$
\left\{H w_{1}, H w_{2}, H w_{3}\right\}=\left\{\lambda_{1} w_{1}, \lambda_{2} w_{2}, \lambda_{3} w_{3}\right\}
$$

для некоторых констант $\lambda_{1}, \lambda_{2}, \lambda_{3}$. Так как $w_{1}+w_{2}+w_{3}=0$, то $H w_{1}+H w_{2}+H w_{3}=$ 0 , то есть $\lambda_{1} w_{1}+\lambda_{2} w_{2}+\lambda_{3} w_{3}=0$. Отсюда, $\lambda_{1}=\lambda_{2}=\lambda_{3}$. Если $H$ поделить на $\lambda_{1}$, то (8) сохранится, а $\gamma_{i k}$ в соответствии с (7) увеличатся в $\lambda_{1}$ раз. Если после этого в алгебре с таблицей умножения (6) все базисные векторы уменьшить в $\lambda_{1}$ раз, то $\gamma_{i k}$ поделятся на $\lambda_{1}$, то есть получается алгебра, изоморфная исходной. Таким образом, достаточно рассматривать только такие матрицы $H$, для которых $\left\{H w_{1}, H w_{2}, H w_{3}\right\}=\left\{w_{1}, w_{2}, w_{3}\right\}$ и выполняется (8). Лемма доказана.

Условиям леммы 7 удовлетворяют только 6 матриц $H$, а именно,

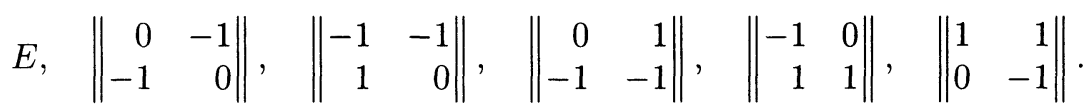


Если $H=E$, то из (8) и (7) получаем, что $\tau$ - тождественная подстановка, $\sigma=\rho$ и $\gamma_{i k}=\operatorname{Tr}\left(S_{k} S_{\rho_{(i)}^{2}}\right)$. Нетрудно проверить, что получается алгебра с таблицей умножения, представленной в табл. 1. Отметим, что строки в табл. 1 переставлены, чтобы добиться большей симметрии. А именно $i$-я строка соответствует произведению $e_{\rho(i)} e_{\rho_{(i)}^{2}}$ и $k$-й элемент в ней равен $\operatorname{Tr}\left(S_{k} S_{i}\right)$. Полученную алгебру обозначим $A M 1$.

Пусть

$$
H=\left\|\begin{array}{rr}
0 & -1 \\
-1 & 0
\end{array}\right\|
$$

Тогда $H w_{1}=w_{2}, H w_{2}=w_{1}, H w_{3}=w_{3}$. При этом легко проверить, что подстановка $\tau$ из (8) имеет вид $\tau=(0)(14)(25)(36)$. Из (7) получаем, что $\sigma=(0)(153426)$ и таблица умножения, вычисленная согласно (6), имеет вид, представленный в табл. 2. Чтобы получить более стандартный вид, заменим в базисе $e_{2}, e_{3}, e_{5}, e_{6}$ на $-e_{2},-e_{3},-e_{5},-e_{6}$. При этом на -1 умножатся строки, соответствующие $e_{1} e_{5}$, $e_{3} e_{4}, e_{4} e_{2}, e_{6} e_{1}$, и столбцы, соответствующие $e_{2}, e_{3}, e_{5}, e_{6}$. Переставляя строки, получим таблицу умножения, представленную в табл. 3. Алгебру с этой таблицей умножения обозначим $A M 2$.

Пусть

$$
H=\left\|\begin{array}{rr}
-1 & -1 \\
1 & 0
\end{array}\right\|, \quad H^{-1}=\left\|\begin{array}{rr}
0 & 1 \\
-1 & -1
\end{array}\right\| .
$$

Тогда $H\left(w_{1}\right)=w_{3}, H\left(w_{2}\right)=w_{1}, H\left(w_{3}\right)=w_{2}$ и подстановка $\tau$ из (8) имеет вид $\tau=(0)(123)(465)$. Из (7) получаем, что $\sigma=(0)(1)(2)(3)(465)$. Вычислим таблицу умножения соответствующей алгебры по формуле (6) и затем заменим в базисе все векторы на противоположные (при этом все $\gamma_{i k}$ умножатся на -1 ). Получающаяся таблица умножения представлена в табл. 4. Соответствующую алгебру обозначим $A M 3$. Докажем теперь следующие утверждения.

Лемма 8. Алгебры $A M 1, A M 2, A M 3$ попарно не изоморфны.

Доказательство. В [1] элемент $x$ алгебры $L$ был назван уникальным, если $\operatorname{dim}(x L) \leqslant 1$, где $x L=\{x a \mid a \in L\}$. Так как никакие две строки в каждой из табл. 1, 3, 4 не пропорциональны, согласно лемме 4 из [1], уникальными векторами в каждой алгебре являются векторы, коллинеарные базисным векторам, и только они. Следовательно, если бы эти алгебры были изоморфны, то при изоморфизме базисные векторы одной алгебры переходили бы в базисные векторы другой алгебры с умножением на некоторые константы. Но тогда подстановки $\sigma$, определяющие ненулевые произведения $e_{i} e_{\sigma(i)}$ в обеих алгебрах, должны были бы быть подобны. Но легко видеть, что наборы длин циклов у подстановок $\sigma$ в алгебрах $A M 1, A M 2, A M 3$ различны. Следовательно, эти алгебры не изоморфны. Лемма доказана.

Обозначим через $A M(H)$ алгебру, построенную в соответствии с леммой 6 для матрицы $H$.

Лемма 9. Пусть $S_{0}, S_{1}, \ldots, S_{6}$ - матрицы Штрассена (2), $\rho=(0)(123)(456) u$ $D$ - матрица, такая, что $D S_{k} D^{-1}=S_{\pi(k)}$, әде $\pi$-некоторая подстановка, 
удовлетворяющая условию $\pi \rho=\rho \pi$. Пусть $H$ и $H_{1}$ - две матрищы из списка (10), $u H_{1}=D H D^{-1}$.

Тогда алгебры $A M(H)$ и $A M\left(H_{1}\right)$ изоморфны.

Доказательство. Так как $H$ и $H_{1}$ входят в список (10),

$$
H S_{k} H^{-1}=S_{\tau(k)}, \quad H_{1} S_{k} H_{1}^{-1}=S_{\tau_{1}} .
$$

Из условий леммы получаем, что

$$
S_{\tau_{1}}=D H D^{-1} S_{k} D H^{-1} D^{-1}=D H_{\pi^{-1}(k)} H^{-1} D^{-1}=D S_{\tau \pi^{-1}(k)} D^{-1}=S_{\pi \tau \pi^{-1}(k)},
$$

то есть $\tau_{1}=\pi \tau \pi^{-1}$. Пусть ненулевые произведения в алгебрах $A M(H)$ и $A M\left(H_{1}\right)$, построенных в соответствии с леммой 6 , задаются подстановками $\sigma$ и $\sigma_{1}$. Тогда

$$
\sigma=\tau^{-1} \rho, \quad \sigma_{1}=\tau_{1}^{-1} \rho=\pi \tau \pi^{-1} \rho .
$$

Таблица умножения в алгебре $A M\left(H_{1}\right)$ имеет вид

$$
e_{i}^{\prime} e_{\sigma_{1}(i)}^{\prime}=\sum_{k=0}^{6} \operatorname{Tr}\left(S_{k} H_{1}^{-1} S_{\rho_{(i)}^{2}}\right) e_{k}^{\prime}
$$

Ho

$$
\begin{aligned}
\operatorname{Tr}\left(S_{k} H_{1}^{-1} S_{\rho_{(i)}^{2}}\right) & =\operatorname{Tr}\left(S_{k} D H^{-1} D^{-1} S_{\rho_{(i)}^{2}}\right)=\operatorname{Tr}\left(D^{-1} S_{k} D H D^{-1} S_{\rho_{(i)}^{2}} D\right) \\
& =\operatorname{Tr}\left(S_{\pi^{-1}(k)} H S_{\pi^{-1} \rho^{2}(i)}\right) .
\end{aligned}
$$

Сделаем замену базиса $e_{i}^{\prime}=e_{\pi^{-1}(i)}$. Тогда получим, что

$$
e_{\pi^{-1}(i)} e_{\pi^{-1} \sigma_{1}(i)}=\sum_{k=0}^{6} \operatorname{Tr}\left(S_{\pi^{-1}(k)} H^{-1} S_{\pi^{-1} \rho^{2}(i)}\right) e_{\pi^{-1}(k)}
$$

Подставив $i=\pi(j), k=\pi(m)$, получим, что

$$
e_{j} e_{\pi^{-1} \sigma_{1} \pi(j)}=\sum_{m=0}^{6} \operatorname{Tr}\left(S_{m} H^{-1} S_{\pi^{-1} \rho^{2} \pi(j)}\right) e_{m} .
$$

Так как $\pi \rho=\rho \pi$, то $\pi^{-1} \sigma_{1} \pi=\tau \pi^{-1} \rho \pi=\tau \rho=\sigma$ и $\pi^{-1} \rho^{2} \pi=\rho^{2}$. Таким образом, таблица умножения в $A M\left(H_{1}\right)$ в новом базисе имеет вид

$$
e_{j} e_{\sigma(j)}=\sum_{m=0}^{6} \operatorname{Tr}\left(S_{m} H^{-1} S_{\rho^{2}(j)}\right) e_{m}
$$

что совпадает с таблицей умножения в алгебре $A M(H)$. Лемма доказана.

Лемма 10. Любая 7-мерная алгебра с простым умножением над полем действительных чисел, являющаяся расширением алгебры матрич порядка 2, изоморфна одной из алгебр $A M 1, A M 2, A M 3$. 
Доказательство. Пусть

$$
D=\left\|\begin{array}{rr}
0 & -1 \\
-1 & 0
\end{array}\right\|
$$

Тогда $D S_{k} D^{-1}=S_{\pi(k)}$, где $\pi=(0)(14)(25)(36)$. При этом $\pi \rho=\rho \pi$. Пусть

$$
H=\left\|\begin{array}{rr}
-1 & -1 \\
1 & 0
\end{array}\right\|
$$

- матрица, задающая алгебру $A M 3$. Рассмотрим матрицу

$$
H_{1}=D H D^{-1}=\left\|\begin{array}{rr}
0 & 1 \\
-1 & -1
\end{array}\right\|
$$

По лемме 9 получаем, что эта матрица задает алгебру, изоморфную $A M 3$.

Пусть

$$
D=\left\|\begin{array}{rr}
-1 & -1 \\
1 & 0
\end{array}\right\|
$$

Тогда $D S_{k} D^{-1}=S_{\pi(k)}$, где $\pi=(0)(123)(465)$, и $\pi \rho=\rho \pi$. Пусть

$$
H=\left\|\begin{array}{rr}
0 & -1 \\
-1 & 0
\end{array}\right\|
$$

- матрица, задающая алгебру $A M 2$. По лемме 9 получаем, что матрицы

$$
H_{1}=D H D^{-1}=\left\|\begin{array}{rr}
-1 & 0 \\
1 & 1
\end{array}\right\|, \quad H_{2}=D H_{1} D^{-1}=\left\|\begin{array}{rr}
1 & 1 \\
0 & -1
\end{array}\right\|
$$

задают алгебры, изоморфные алгебре $A M 2$.

Рассмотренные алгебры $H$ исчерпывают весь список (10), поэтому утверждение леммы 10 вытекает из лемм 6 и 7.

Леммы 8 и 10 вместе составляют утверждение доказываемой теоремы. Таким образом, теорема полностью доказана.

Один из интересных открытых вопросов -- найти алгебру с простым умножением минимальной размерности, являющуюся расширением алгебры матриц порядка $n$, что явилось бы существенным продвижением в задаче о сложности умножения матриц. Для начала было бы интересно получить решение для случая $n=3$. Доказанная теорема может помочь в этом поиске.

\section{Список литературы}

1. Алексеев В. Б. О некоторых алгебрах, связанных с быстрыми алгоритмами. Дискретная математика (1996) 8, №1, 52-64.

2. Штрассен В. Алгоритм Гаусса не оптимален. Киберн. сб. (1970) 7, 67-70.

3. de Groote H. F. On varieties of optimal algorithms for the computation of bilinear mappings. II. Optimal algorithms for $2 \times 2$ matrix multiplication. Theoretical Comput. Sci. (1978) 7, №.2, 127-148.

4. Алексеев В. Б. Сложность умножения матриц. Обзор. Киберн. сб. (1988) 25, 189-236. 